\title{
Non-pharmacological Treatment for Chronic Pain in US Veterans Treated Within the Veterans Health Administration: Implications for Expansion in US Healthcare Systems
}

\author{
Zachary L. Mannes, PhD, MPH ${ }^{1,2}$, Malki Stohl, MS' , David S. Fink, PhD, MPH², \\ Mark Olfson, $M D, M P H^{3}$, Katherine M. Keyes, $P h D, M P H^{7}$, Silvia S. Martins, $M D, P h D^{7}$, \\ Jaimie L. Gradus, DSC, MPH4,5, Andrew J. Saxon, MD ${ }^{6,7}$, Charles Maynard, $P h D^{6,7}$, \\ Ofir Livne, $\mathrm{MD}^{1,2}$, Sarah Gutkind, $\mathrm{MSPH}^{7}$, and Deborah S. Hasin, PhD
}

\begin{abstract}
'Department of Epidemiology, Columbia University Mailman School of Public Health, New York, NY, USA; ${ }^{2}$ New York State Psychiatric Institute, 1051 Riverside Drive, New York, NY, USA; ${ }^{3}$ Department of Psychiatry, Columbia University Irving Medical Center, New York, NY, USA; ${ }^{4}$ Department of Epidemiology, Boston University School of Public Health, Boston, MA, USA; ${ }^{5}$ Department of Psychiatry, Boston University School of Medicine, Boston, MA, USA; 'Department of Psychiatry \& Behavioral Sciences, University of Washington School of Medicine, Seattle, WA, USA; ${ }^{6}$ VA Puget Sound Healthcare System, Seattle, WA, USA.
\end{abstract}

BACKGROUND: Consensus guidelines recommend multimodal chronic pain treatment with increased use of nonpharmacological treatment modalities (NPM), including as first-line therapies. However, with many barriers to NPM uptake in US healthcare systems, NPM use may vary across medical care settings. Military veterans are disproportionately affected by chronic pain. Many veterans receive treatment through the Veterans Health Administration (VHA), an integrated healthcare system in which specific policies promote NPM use.

OBJECTIVE: To examine whether veterans with chronic pain who utilize VHA healthcare were more likely to use NPM than veterans who do not utilize VHA healthcare.

DESIGN: Cross-sectional nationally representative study. PARTICIPANTS: US military veterans $(N=2,836)$.

MAIN MEASURES: In the 2019 National Health Interview Survey, veterans were assessed for VHA treatment, chronic pain (i.e., past 3-month daily or almost daily pain), symptoms of depression and anxiety, substance use, and NPM (i.e., physical therapy, chiropractic/spinal manipulation, massage, psychotherapy, educational class/ workshop, peer support groups, or yoga/tai chi).

KEY RESULTS: Chronic pain (45.2\% vs. $26.8 \%$ ) and NPM use $(49.8 \%$ vs. $39.4 \%)$ were more prevalent among VHA patients than non-VHA veterans. After adjusting for sociodemographic characteristics, psychiatric symptoms, physical health indicators, and use of cigarettes or prescription opioids, VHA patients were more likely than non-VHA veterans to use any NPM (adjusted odds ratio [aOR] $=1.52,95 \%$ CI: 1.07-2.16) and multimodal NPM $(\mathrm{aOR}=1.80,95 \% \mathrm{CI}: 1.12-2.87)$ than no NPM. Among veterans with chronic pain, VHA patients were more likely to use chiropractic care $(\mathrm{aOR}=1.90,95 \% \mathrm{CI}=1.12-3.22)$, educational class/workshop $(\mathrm{aOR}=3.02$, 95\% CI $=1.35$ $6.73)$, or psychotherapy $(\mathrm{aOR}=4.28,95 \% \mathrm{CI}=1.69$ 10.87).

Prior Presentations None

Received September 23, 2021

Accepted December 16, 2021

Published online January 19, 2022
CONCLUSIONS: Among veterans with chronic pain, pastyear VHA use was associated with greater likelihood of receiving NPM. These findings may suggest that the VHA is an important resource and possible facilitator of NPM. VHA policies may offer guidance for expanding use of NPM in other integrated US healthcare systems.

KEY WORDS: chronic pain; prescription opioid use; non-pharmacological pain treatment; military veterans; Veterans Health Administration.

J Gen Intern Med 37(15):3937-46

DOI: $10.1007 / \mathrm{s} 11606-021-07370-8$

(c) The Author(s) 2022

\section{INTRODUCTION}

Chronic pain, i.e., pain that persists or recurs for 3 months or more ${ }^{1,2}$, affects $20 \%$ of US adults, ${ }^{3}$ contributes to an annual economic cost of $\$ 635$ billion $^{4}$, and is associated with disability, cognitive impairment, cardiovascular disease, and psychiatric and substance use disorders. ${ }^{5-8}$ Opioid prescribing was previously considered standard of care for chronic pain, ${ }^{9}$ and the national opioid dispensing rate increased from 1999 to 2012, accompanied by increases in morbidity and mortality. ${ }^{10,11}$ After the risks of excessive prescription of opioids became widely recognized, rates of opioid prescribing declined ${ }^{12}$ and the Centers for Disease Control and Prevention (CDC) began recommending the use of non-pharmacological modalities (NPM) such as physical rehabilitation, chiropractic care, and psychological treatments as first-line alternatives to opioids for chronic pain. ${ }^{13}$ Use of NPM, particularly multimodal treatment (i.e., concurrent use of $\geq 2$ NPM), can yield improvements in pain, functioning, and quality of life, ${ }^{14}$, 15 is associated with lower healthcare costs, ${ }^{16}$ and may reduce receipt of prescription opioids. ${ }^{17}$ However, high cost, limited provider reimbursement, and poor access, or the inability to obtain affordable, convenient healthcare 
services, ${ }^{18}$ has limited use of NPM within US healthcare settings. ${ }^{19-23}$ With decreasing rates of opioid prescribing in the US, ${ }^{12}$ patients with chronic pain may be left untreated unless alternative therapies are provided. Therefore, identifying healthcare settings where patients are likely to use NPM, or those where NPM resources are less available, may guide initiatives aimed at improving access to these treatments, particularly among populations vulnerable to chronic pain.

Chronic pain is particularly prevalent among US military veterans, affecting approximately one-third of veterans receiving healthcare through the Veterans Health Administration (VHA). ${ }^{24,}{ }^{25}$ Opioid prescribing in the VHA increased from 17 to $24 \%$ between 2001 and $2009,{ }^{26}$ and was associated with opioid-related hospitalizations, overdose, and self-inflicted injuries. $^{27,} 28$ These harms were highest among veterans with depression and anxiety disorders, ${ }^{28}$ which are more prevalent in veterans than the US general population. ${ }^{29}$ To mitigate these consequences, the VHA enacted the 2013 Opioid Safety Initiative, a multimodal clinical program that contributed to a $64 \%$ decrease in opioid prescribing. ${ }^{30,31}$ Consistent with CDC guidelines, the VHA now emphasizes use of NPM, ${ }^{32}$ which is associated with lower risk of overdose, suicidal ideation, and self-injurious behavior. ${ }^{33}$

The VHA is the largest integrated healthcare system in the US, providing healthcare to over 5.5 million veterans annually, including chronic pain services without the need for outside referrals or prior authorizations. ${ }^{34}$ The VHA also promotes a holistic approach to chronic pain treatment $^{35,36}$ through the Whole Health Program, a wellness initiative leveraging complimentary and integrative health services. ${ }^{37}$ However, not all veterans are eligible for VHA services due to not meeting eligibility requirements such as low income or service-related disabilities, and $17 \%$ of veterans who are enrolled in the VHA use it as their only source of care. ${ }^{38,39}$ Consequently, use of NPM may depend on whether veterans receive their care in the VHA, where some barriers remain although concerted efforts are in place to overcome them, ${ }^{19,20,40,41}$ or in other healthcare systems with persisting obstacles to NPM provision. ${ }^{21-23}$ In this study, we investigated whether veterans with chronic pain who received their care within the VHA's integrated system were more or less likely to use NPM than veterans outside the VHA. Drawing on data from the 2019 National Health Interview Survey (NHIS), we first examined differences in the prevalence of chronic pain between veterans receiving their healthcare from the VHA and those who do not, as these findings may have important implications for NPM resource allocation. Second, we examined associations between VHA healthcare use and NPM among veterans with chronic pain. We hypothesized that among veterans with chronic pain, VHA patients would be more likely to use NPM than non-VHA veterans.

\section{METHODS}

\section{Sample and Design}

Data were derived from the 2019 NHIS Adult component (https://www.cdc.gov/nchs/nhis/2019nhis.htm; $N=31,997$ ), a yearly health survey that characterizes the self-reported physical and mental health of the non-institutionalized civilian population of the US, including military veterans. Trained interviewers collected NHIS data from January to December, 2019. The 2019 NHIS adult response rate was 59.1\%. The analytic sample for this report consisted of participants who self-reported a history of military service and at least one healthcare visit within the prior 12 months $(N=2,836)$, of whom 10 were excluded due to missing age information, yielding an analytic sample of 2,826 respondents. The New York State Psychiatric Institute's Institutional Review Board did not require human subjects review as we analyzed deidentified, public-use data.

\section{Measures}

Chronic Pain andHigh-Impact Chronic Pain. Participants were asked, "In the past three months, how often did you have pain? Would you say never, some days, most days, or every day?" We defined chronic pain as pain "most days" or "every day"., 2 Participants were then asked, "Over the past three months, how often did your pain limit your life or work activities? Would you say never, some days, most days, or every day?" High-impact chronic pain was defined as pain that limited life or work activities most days or every day. These definitions have been used in other NHIS studies ${ }^{3,42-45}$ and to assess pain among veterans. ${ }^{24-26}$

Exposure. VHA Healthcare. Most VHA patients utilize care at VHA facilities, although the VHA also covers care by designated community clinicians when it cannot provide needed care. ${ }^{38}$ Veterans were asked: "During the past 12 months, did you receive any care at a Veteran's Health Administration facility or receive any other health care paid for by the VA?" Interviewers prompted participants that VHA care included VA hospitals, medical centers, outpatient clinics, and nursing homes. A dichotomous variable assessed past 12-month use of VHA healthcare (labeled hereafter as "VHA patients" and "non-VHA veterans)."

Outcome. NPM. The VHA recommends several evidencebased NPM. ${ }^{46}$ Participants were asked about their NPM use for pain with the follow question: "Over the past three months, did you use any of the following to manage your pain?" NPM included physical therapies (physical, rehabilitative, or occupational therapy); chiropractic/spinal manipulation; massage; psychotherapy; educational class/workshop; peer support groups; yoga/tai chi; or meditation/guided imagery/other relaxation techniques. Participants were not asked where they 
Table 1 Characteristics of study sample $(N=\mathbf{2 , 8 3 6})$

\begin{tabular}{|c|c|c|c|c|}
\hline \multirow[t]{2}{*}{ Variable } & \multirow{2}{*}{$\begin{array}{l}\text { VHA patients } \\
(N=983) \\
\% \text { (SE) }\end{array}$} & \multirow{2}{*}{$\begin{array}{l}\text { Non-VHA veterans } \\
(N=1,853) \\
\%(\mathrm{SE})\end{array}$} & \multirow{2}{*}{$\begin{array}{l}\text { All veterans } \\
(N=\mathbf{2 , 8 3 6 )} \\
\%(\mathrm{SE})\end{array}$} & \multirow[b]{2}{*}{$p$ value } \\
\hline & & & & \\
\hline Age & & & & 0.32 \\
\hline 18-34 & $11.25(1.41)$ & $8.59(1.01)$ & $9.47(0.85)$ & \\
\hline $35-44$ & $9.33(1.08)$ & $8.54(0.81)$ & $8.80(0.68)$ & \\
\hline $45-54$ & $11.54(1.36)$ & $14.09(1.07)$ & $13.25(0.84)$ & \\
\hline $55-64$ & $17.32(1.36)$ & $18.61(1.06)$ & $18.19(0.82)$ & \\
\hline$\geq 65$ & $50.55(2.02)$ & $50.17(1.40)$ & $50.30(1.17)$ & \\
\hline $\operatorname{Sex}$ & & & & 0.68 \\
\hline Men & $89.55(1.22)$ & $88.91(0.87)$ & $89.12(0.67)$ & \\
\hline Women & $10.45(1.22)$ & $11.09(0.87)$ & $10.88(0.67)$ & \\
\hline Race/ethnicity & & & & $<0.01$ \\
\hline Non-Hispanic, White & $70.86(1.99)$ & $82.59(1.25)$ & $78.73(1.13)$ & \\
\hline Non-Hispanic, Black & $18.05(1.67)$ & $8.79(0.94)$ & $11.84(0.86)$ & \\
\hline Non-Hispanic, Asian & $2.39(0.80)$ & $1.30(0.33)$ & $1.66(0.34)$ & \\
\hline Hispanic & $6.56(0.88)$ & $4.66(0.68)$ & $5.28(0.53)$ & \\
\hline Other $^{a}$ & $2.14(0.50)$ & $2.67(0.48)$ & $2.49(0.38)$ & \\
\hline Education & & & & 0.33 \\
\hline High school & $32.25(1.88)$ & $30.05(1.36)$ & $30.77(1.14)$ & \\
\hline Some college or more & $67.75(1.88)$ & $69.95(1.36)$ & $69.23(1.14)$ & \\
\hline Family income (FPL) & & & & $<0.001$ \\
\hline$<100 \%$ & $6.07(0.92)$ & $4.07(0.56)$ & $4.73(0.49)$ & \\
\hline $100-199 \%$ & $19.36(1.43)$ & $11.82(0.97)$ & $14.30(0.79)$ & \\
\hline $200-399 \%$ & 38.98 (1.87) & $33.71(1.30)$ & $35.45(1.06)$ & \\
\hline$\geq 400 \%$ & $35.58(1.82)$ & $50.40(1.47)$ & $45.52(1.20)$ & \\
\hline $\mathbf{U} \overline{\mathbf{S}}$ region & & & & 0.44 \\
\hline Northeast & $14.83(1.79)$ & $17.29(1.12)$ & $16.48(1.04)$ & \\
\hline Midwest & $21.00(1.59)$ & $21.05(1.31)$ & $21.03(1.09)$ & \\
\hline South & $44.61(2.11)$ & $41.29(1.66)$ & $42.38(1.44)$ & \\
\hline West & $19.57(1.71)$ & $20.37(1.28)$ & $20.11(1.12)$ & \\
\hline Urbanicity $^{\mathbf{b}}$ & & & & 0.14 \\
\hline Metropolitan & $80.59(1.65)$ & $83.21(1.19)$ & $82.34(1.06)$ & \\
\hline GAD symptoms ${ }^{\mathrm{c}}$ & & & & $<0.01$ \\
\hline Moderate/severe & $10.16(1.09)$ & $3.40(0.51)$ & $5.63(0.49)$ & \\
\hline MDD symptoms ${ }^{\mathrm{d}}$ & & & & $<0.01$ \\
\hline $\begin{array}{l}\text { Moderate/severe } \\
\text { Cigarette use }\end{array}$ & $12.46(1.26)$ & $6.10(0.81)$ & $8.20(0.68)$ & \\
\hline $\begin{array}{l}\text { Cigarette use }{ }^{\mathbf{e}} \\
\text { Current smoker }\end{array}$ & & & & 0.08 \\
\hline $\begin{array}{l}\text { Current smoker } \\
\text { BMI }^{\mathbf{f}}\end{array}$ & $17.76(1.40)$ & $14.68(1.16)$ & $15.69(0.91)$ & 023 \\
\hline $\begin{array}{l}\text { BMI }^{\mathbf{T}} \\
\text { Overweight or obese }^{-1}\end{array}$ & $77.22(1.50)$ & & $75.68(0.99)$ & 0.23 \\
\hline Chronic pain ${ }^{\mathrm{g}}$ & $45.26(1.90)$ & $26.88(1.21)$ & $32.93(1.07)$ & $<0.01$ \\
\hline $\begin{array}{l}\text { High-impact } \\
\text { chronic pain }\end{array}$ & $26.04(1.99)$ & $15.20(1.24)$ & $19.15(1.10)$ & $<0.01$ \\
\hline
\end{tabular}

Note. Bold values indicate significance at $p<0.05$. VHA Veterans Health Administration, FPL federal poverty line, GAD generalized anxiety disorder, $M D D$ major depressive disorder, BMI body mass index

${ }^{a}$ Included biracial adults

${ }^{b}$ Metropolitan defined as large central, fringe, medium, or small metropolitan vs. non-metropolitan

${ }^{c}$ Generalized Anxiety Disorder Scale-7 item (GAD-7) assessed generalized anxiety disorder symptoms during the past 2 weeks. Participants were categorized into none to mild (0-9) symptoms and moderate to severe symptoms (10-21)

${ }^{d}$ Patient Health Questionnaire Depression Scale-8 item (PHQ-8) assessed depressive symptoms during the past 2 weeks. Participants were categorized into none to mild (0-9) and moderate to severe (15-24) symptom severity

eIncludes combustible or electronic cigarette use

${ }^{f}$ Overweight or obese vs. underweight or healthy weight

${ }^{g}$ Pain every day or most days during the past 3 months

${ }^{h}$ Chronic pain that interferes with daily activities every day or on most days during the past 3 months

obtained these services, but they could endorse use of multiple NPM. Dichotomous variables were created for each treatment modality (use; no use). We also created variables indicating use of $\geq 1 \mathrm{NPM}$ and $\geq 2 \mathrm{NPM}$, since multimodal pain treatment is associated with improved health outcomes. ${ }^{14,15}$

Covariates. Prescription Opioid Use. Participants were asked: "During the past 3 months, did you take a prescription opioid to treat long-term or chronic pain, such as low back pain or neck pain, frequent headaches or migraines, or joint pain or arthritis?" A dichotomous (yes; no) variable indicated past 3month prescription opioid use for chronic pain.

Depressive Symptoms. The Patient Health Questionnaire (PHQ-8) assessed depression symptoms over the past 2 weeks with eight Likert scale items ranging from " 0 " (not at all) to "3" (nearly every day). Scores were dichotomized into no/mild symptoms (score 0-9), and moderate/severe symptoms (score $\geq 10) .{ }^{47}$ The PHQ-8 has strong sensitivity and specificity for major depressive disorder. ${ }^{47}$ 
Anxiety Symptoms. The Generalized Anxiety Disorder 7-item Assessment (GAD-7) measured anxiety over the past 2 weeks with seven Likert scale items ranging from "0" (not at all) to "3" (nearly every day). Participants were dichotomized into none/mild symptoms (score 0-9), and moderate/severe symptoms (score $\geq 10$ ). ${ }^{48}$ The GAD-7 has high internal and external reliability. ${ }^{48}$

Cigarette Use. Current smokers (past 30-day use), former smokers (use more than 30 days ago), and never smokers were categorized into three, mutually exclusive groups. Cigarette use is associated with chronic pain ${ }^{49}$ and healthcare utilization $^{50}$ among VHA patients.

Body Mass Index (BMI). BMI was calculated using selfreported height and weight. Due to low prevalence of underweight in this sample $(1.61 \%)$, participants were categorized into two groups: underweight and healthy weight (BMI < $24.9)$ vs. overweight or obese (BMI $\geq 25$ ). Overweight and obesity are associated with chronic pain and other morbidities contributing to use of healthcare services. ${ }^{51}$

Sociodemographic Characteristics. Age (18-34, 35-49, 50$64, \geq 65$ ), sex (male, female), race/ethnicity (non-Hispanic/ White, non-Hispanic/Black, non-Hispanic/Asian, Hispanic/Latino, other), education (high school or equivalent, college or more), family income relative to the federal poverty line (FPL; $<100 \%$ FPL, 100-199\%, 200-399\% FPL, $\geq 400 \%$ FPL), US region of residence (Northeast, Midwest, South, West), and county urbanicity (large central metropolitan, large fringe metropolitan, medium/small metropolitan, nonmetropolitan). ${ }^{52}$

\section{Statistical Analysis}

Analyses were conducted using SAS-callable SUDAAN 11.0.1 and were weighted and adjusted for the complex survey design. Among all veterans, chi-square tests assessed differences in prevalence of sociodemographic characteristics, psychiatric symptoms, cigarette use, chronic pain, and high-impact chronic pain by VHA utilizer status. Among veterans with chronic pain, chisquare tests assessed differences in past 3-month use of prescription opioids or NPM among VHA and non-VHA veterans. Binary logistic regression models were then run to examine associations between VHA utilization and use of any NPM ( $\geq 1$ vs. 0 ) or use of each NPM (yes vs. no) adjusting for age, sex, race/ethnicity, education, income, US region, urbanicity, BMI, generalized anxiety or depressive symptoms, high-impact chronic pain, and use of cigarettes or prescription opioids. A multinomial logistic regression model was also fit to examine the association between VHA utilization with a three-level NPM use outcome (1 NPM, $\geq 2 \mathrm{NPM,} \mathrm{vs.} \mathrm{0)} \mathrm{adjusting} \mathrm{for}$ covariates, from which adjusted odds ratios (aOR) with 95\% confidence intervals (CIs) were derived.

\section{RESULTS}

\section{Sample Characteristics}

Veterans were primarily male (89.1\%), non-Hispanic, White $(78.7 \%)$, with at least some college education $(69.2 \%)$, and many were aged 65 or older (50.3\%), had family income $\geq$ $400 \%$ FPL $(45.5 \%)$, and resided in metropolitan areas $(82.3 \%)$. Approximately $35 \%$ of veterans used the VHA

Table 2 Prevalence of pain treatment modalities among veterans with chronic pain $(N=936)^{a}$

\begin{tabular}{|c|c|c|c|c|}
\hline \multirow[t]{2}{*}{ Variable } & \multirow{2}{*}{$\begin{array}{l}\text { VHA patients } \\
(N=445) \\
\% \text { (SE) }\end{array}$} & \multirow{2}{*}{$\begin{array}{l}\text { Non-VHA veterans } \\
(N=491) \\
\%(\mathrm{SE})\end{array}$} & \multirow{2}{*}{$\begin{array}{l}\begin{array}{l}\text { All veterans } \\
(N=936)\end{array} \\
\%(\mathrm{SE})\end{array}$} & \multirow[b]{2}{*}{$p$ value } \\
\hline & & & & \\
\hline Opioid use ${ }^{b}$ & $13.84(1.71)$ & $15.86(2.17)$ & $14.94(1.39)$ & 0.479 \\
\hline \multicolumn{5}{|l|}{$\mathrm{NPM}^{\mathrm{c}}$} \\
\hline Physical therapies & $24.48(2.46)$ & $18.30(1.93)$ & $21.10(1.55)$ & 0.04 \\
\hline Chiropractic care & $14.11(1.91)$ & $9.32(1.65)$ & $11.49(1.23)$ & 0.06 \\
\hline Massage & $15.42(2.06)$ & $15.29(2.16)$ & $15.34(1.43)$ & 0.96 \\
\hline Psychotherapy & $7.13(1.65)$ & $1.59(0.59)$ & $4.09(0.82)$ & $<0.01$ \\
\hline Education class/workshop & $8.36(1.54)$ & $3.54(0.89)$ & $5.72(0.85)$ & $<0.01$ \\
\hline Peer support group & $2.65(1.06)$ & $0.78(0.36)$ & $1.63(0.52)$ & 0.09 \\
\hline Yoga/tai chi & $7.24(1.32)$ & $4.99(1.31)$ & $6.01(1.03)$ & 0.17 \\
\hline Meditation/relaxation techniques & $16.05(2.02)$ & $11.09(1.69)$ & $13.34(1.29)$ & 0.06 \\
\hline \multicolumn{5}{|l|}{ NPM } \\
\hline Yes $(\geq 1)$ & $49.83(2.82)$ & $39.48(2.66)$ & $43.80(2.01)$ & $<0.01$ \\
\hline Total number of NPM & & & & 0.01 \\
\hline 0 & $50.17(2.82)$ & $60.52(2.66)$ & $55.84(2.01)$ & \\
\hline 1 & $26.59(2.64)$ & $23.02(2.23)$ & $24.63(1.76)$ & \\
\hline$\geq 2$ & $23.23(2.41)$ & $16.46(2.14)$ & $19.53(1.61)$ & \\
\hline
\end{tabular}

Note. Bold values indicate significance at $p<0.05$. VHA Veterans Health Administration, NPMnon-pharmacological treatment modalities

${ }^{a}$ Includes 936 participants reporting daily or almost daily pain over the past 3 months

${ }^{b}$ Past 3-month prescription opioid use for chronic pain

${ }^{c}$ Past 3-month pain treatment (yes, no) 
Table 3 Association of VHA utilization and any NPM use among veterans with chronic pain $(N=936)$

\begin{tabular}{|c|c|}
\hline \multirow[t]{2}{*}{$\overline{\text { Variable }}$} & \multirow{2}{*}{$\begin{array}{l}\geq 1 \text { NPM }(n=410) \\
(\text { vs. no NPM) } \\
\text { AOR }^{\text {a }} \\
(95 \% \text { CI })\end{array}$} \\
\hline & \\
\hline \multicolumn{2}{|l|}{ VHA utilizer status } \\
\hline Non-VHA veteran & ref \\
\hline VHA patient & $1.52(1.07,2.16)$ \\
\hline \multicolumn{2}{|l|}{ Age } \\
\hline $18-34$ & ref \\
\hline $35-44$ & $0.77(0.31,1.90)$ \\
\hline $45-54$ & $0.46(0.21,1.01)$ \\
\hline $55-64$ & $0.36(0.17,0.78)$ \\
\hline$\geq 65$ & $0.26(0.13,0.54)$ \\
\hline \multicolumn{2}{|r|}{ 0. } \\
\hline Male & ref \\
\hline Female & $1.60(0.94,2.72)$ \\
\hline \multicolumn{2}{|l|}{ Race/ethnicity } \\
\hline Non-Hispanic, White & ref \\
\hline Non-Hispanic, Black & $0.70(0.38,1.29)$ \\
\hline Non-Hispanic, Asian & $0.80(0.14,4.54)$ \\
\hline Hispanic & $1.00(0.36,2.78)$ \\
\hline Other ${ }^{\mathrm{b}}$ & $1.25(0.56,2.78)$ \\
\hline \multicolumn{2}{|l|}{ Education } \\
\hline High school & ref \\
\hline Some college or more & $2.07(1.42,3.01)$ \\
\hline \multicolumn{2}{|l|}{ Family income } \\
\hline$<100 \%$ FPL & ref \\
\hline $100-199 \%$ FPL & $1.00(0.47,2.13)$ \\
\hline $200-399 \%$ FPL & $1.29(0.68,2.44)$ \\
\hline$\geq 400 \%$ FPL & $1.70(0.88,3.30)$ \\
\hline \multicolumn{2}{|l|}{ US region } \\
\hline Northeast & ref \\
\hline Midwest & $1.00(0.55,1.79)$ \\
\hline South & $0.86(0.51,1.46)$ \\
\hline West & $1.09(0.63,1.90)$ \\
\hline \multicolumn{2}{|l|}{ GAD symptoms ${ }^{c}$} \\
\hline None/mild & \\
\hline Moderate/severe & $1.41(0.65,3.07)$ \\
\hline \multicolumn{2}{|l|}{ MDD symptoms ${ }^{\mathrm{d}}$} \\
\hline None/mild & ref \\
\hline Moderate/severe & $0.84(0.42,1.71)$ \\
\hline \multicolumn{2}{|l|}{ Cigarette use $^{\mathrm{e}}$} \\
\hline Never & ref \\
\hline Former & $0.85(0.57,1.28)$ \\
\hline \multirow{2}{*}{\multicolumn{2}{|c|}{ Opioid use ${ }^{f}$}} \\
\hline & \\
\hline & \\
\hline Yes & $1.60(0.97,2.65)$ \\
\hline \multicolumn{2}{|l|}{ High-impact chronic paing } \\
\hline No & ref \\
\hline \multirow{2}{*}{\multicolumn{2}{|c|}{$1.60(1.13,2.25)$}} \\
\hline & \\
\hline Underweight or normal weight & ref \\
\hline Overweight or obese & $0.74(0.48,1.14)$ \\
\hline \multicolumn{2}{|l|}{ Urbanicity $^{h}$} \\
\hline Nonmetropolitan & ref \\
\hline Metropolitan & $1.26(0.82,1.91)$ \\
\hline
\end{tabular}

Note. Bold values indicate $p<0.05$. aOR adjusted odds ratio, NPMnon-pharmacological treatment modalities, VHA Veterans Health Administration, FPL federal poverty line, GAD generalized anxiety disorder, MDD major depressive disorder, BMI body mass index

${ }^{a}$ Model adjusted for age, sex, racelethnicity, education, family income, US region, GAD, MDD, and use of cigarettes or prescription opioids, highimpact chronic pain, BMI, and urbanicity

${ }^{b}$ Included biracial adults

${ }^{c}$ Generalized Anxiety Disorder Scale-7 item (GAD-7) assessed generalized anxiety disorder symptoms during the past 2 weeks. Participants were categorized into none to mild (0-9) symptoms and moderate to severe symptoms (10-21)

${ }^{d}$ Patient Health Questionnaire Depression Scale-8 item (PHQ-8) assessed depressive symptoms during the past 2 weeks. Participants were categorized into none to mild (0-9) and moderate to severe (15-24) symptom severity

${ }^{e}$ Includes combustible or electronic cigarette use

${ }^{f}$ Past 3-month prescription opioid use for chronic pain

${ }^{g}$ Chronic pain that interferes with daily activities every day or on most days during the past 3 months

${ }^{h}$ Metropolitan defined as large central, fringe, medium, or small metropolitan 
Table 4 Association of VHA utilization with use of 1 or $\geq 2$ NPM among veterans with chronic pain $(N=936)$

\begin{tabular}{|c|c|c|}
\hline \multirow[t]{2}{*}{ Variable } & \multirow{2}{*}{$\begin{array}{l}1 \mathrm{NPM} \\
(\mathrm{vs} . \mathrm{no} \mathrm{NPM} ; n=231) \\
\mathrm{AOR}^{\mathrm{a}} \\
(95 \% \mathrm{CI})\end{array}$} & \multirow{2}{*}{$\begin{array}{l}\geq 2 \text { NPM } \\
\text { (vs. no NPM; } n=179) \\
\text { AOR }^{\mathrm{a}} \\
(95 \% \mathrm{CI})\end{array}$} \\
\hline & & \\
\hline \multicolumn{3}{|l|}{ VHA utilizer status } \\
\hline Non-VHA veteran & ref & \\
\hline VHA patient & $1.36(0.91,2.03)$ & $1.80(1.12,2.87)$ \\
\hline \multicolumn{3}{|l|}{ Age } \\
\hline $18-34$ & ref & ref \\
\hline $35-44$ & $0.91(0.28,3.01)$ & $0.70(0.25,2.01)$ \\
\hline $45-54$ & $0.68(0.23,2.00)$ & $0.31(0.12,0.80)$ \\
\hline $55-64$ & $0.52(0.18,1.50)$ & $0.24(0.10,0.60)$ \\
\hline \multirow{2}{*}{\multicolumn{3}{|c|}{$0.10(0.0 /, 0.0 /)$}} \\
\hline & & \\
\hline Male & ref & ref \\
\hline Female & $1.08(0.55,2.14)$ & $2.21(1.17,4.19)$ \\
\hline \multicolumn{3}{|l|}{ Race/ethnicity } \\
\hline Non-Hispanic, White & ref & ref \\
\hline Non-Hispanic, Black & $0.80(0.41,1.59)$ & $0.56(0.25,1.26)$ \\
\hline Non-Hispanic, Asian & $0.65(0.09,4.73)$ & $0.81(0.11,5.81)$ \\
\hline Hispanic & $1.43(0.48,4.27)$ & $0.62(0.18,2.09)$ \\
\hline Other ${ }^{b}$ & $0.83(0.32,2.18)$ & $1.79(0.67,4.79)$ \\
\hline \multicolumn{3}{|l|}{ Education } \\
\hline High school & ref & ref \\
\hline \multirow{2}{*}{\multicolumn{3}{|c|}{ Family income }} \\
\hline & & \\
\hline$<100 \%$ FPL & ref & ref \\
\hline $100-199 \%$ FPL & $1.06(0.41,2.73)$ & $0.92(0.35,2.40)$ \\
\hline $200-399 \%$ FPL & $1.43(0.61,3.35)$ & $1.10(0.46,2.59)$ \\
\hline$\geq 400 \%$ FPL & $1.57(0.66,3.77)$ & $1.91(0.84,4.34)$ \\
\hline \multicolumn{3}{|l|}{ US region } \\
\hline Northeast & ref & ref \\
\hline Midwest & $1.01(0.51,1.99)$ & $0.98(0.44,2.17)$ \\
\hline South & $0.85(0.46,1.56)$ & $0.90(0.45,1.80)$ \\
\hline West & $0.81(0.43,1.53)$ & $1.61(0.79,3.27)$ \\
\hline \multicolumn{3}{|l|}{ GAD symptoms ${ }^{\mathrm{c}}$} \\
\hline None/mild & ref & ref \\
\hline Moderate/severe & $1.81(0.75,4.36)$ & $1.09(0.44,2.73)$ \\
\hline \multicolumn{3}{|l|}{ MDD symptoms } \\
\hline None/mild & & ref \\
\hline \multirow{2}{*}{\multicolumn{3}{|c|}{ Cigarette use $\mathrm{e}^{\mathrm{e}}$}} \\
\hline & & \\
\hline Never & ref & ref \\
\hline Former & $1.04(0.66,1.65)$ & $0.64(0.38,1.06)$ \\
\hline \multirow{2}{*}{\multicolumn{3}{|c|}{ Opioid use ${ }^{f}$}} \\
\hline & & \\
\hline No & & ref \\
\hline \multirow{2}{*}{\multicolumn{3}{|c|}{ High-impact chronic paing }} \\
\hline & & \\
\hline No & ref & ref \\
\hline Yes & $1.40(0.94,2.09)$ & $1.95(1.22,2.13)$ \\
\hline \multicolumn{3}{|r|}{$1.70(1.22,2.10)$} \\
\hline Underweight or normal weight & ref & ref \\
\hline Overweight or obese & $0.73(0.45,1.20)$ & $0.78(0.45,1.37)$ \\
\hline \multicolumn{3}{|l|}{ Urbanicity } \\
\hline Nonmetropolitan & ref & ref \\
\hline Metropolitan & $1.28(0.81,2.02)$ & $1.22(0.64,2.33)$ \\
\hline
\end{tabular}

Note. Bold values indicate $p<0.05$. aOR adjusted odds ratio, NPMnon-pharmacological treatment modalities, VHA Veterans Health Administration, FPL federal poverty line, GAD generalized anxiety disorder, MDD major depressive disorder, BMI body mass index

${ }^{a}$ Model adjusted for age, sex, racelethnicity, education, family income, US region, GAD, MDD, and use of cigarettes or prescription opioids, highimpact chronic pain, BMI, and urbanicity

${ }^{b}$ Included biracial adults

${ }^{c}$ Generalized Anxiety Disorder Scale-7 item (GAD-7) assessed generalized anxiety disorder symptoms during the past 2 weeks. Participants were categorized into none to mild (0-9) symptoms and moderate to severe symptoms (10-21)

${ }^{d}$ Patient Health Questionnaire Depression Scale-8 item (PHQ-8) assessed depressive symptoms during the past 2 weeks. Participants were categorized into none to mild (0-9) and moderate to severe (15-24) symptom severity

${ }^{e}$ Includes combustible or electronic cigarette use

${ }^{f}$ Past 3-month prescription opioid use for chronic pain

${ }^{g}$ Chronic pain that interferes with daily activities every day or on most days during the past 3 months

${ }^{h}$ Metropolitan defined as large central, fringe, medium, or small metropolitan 


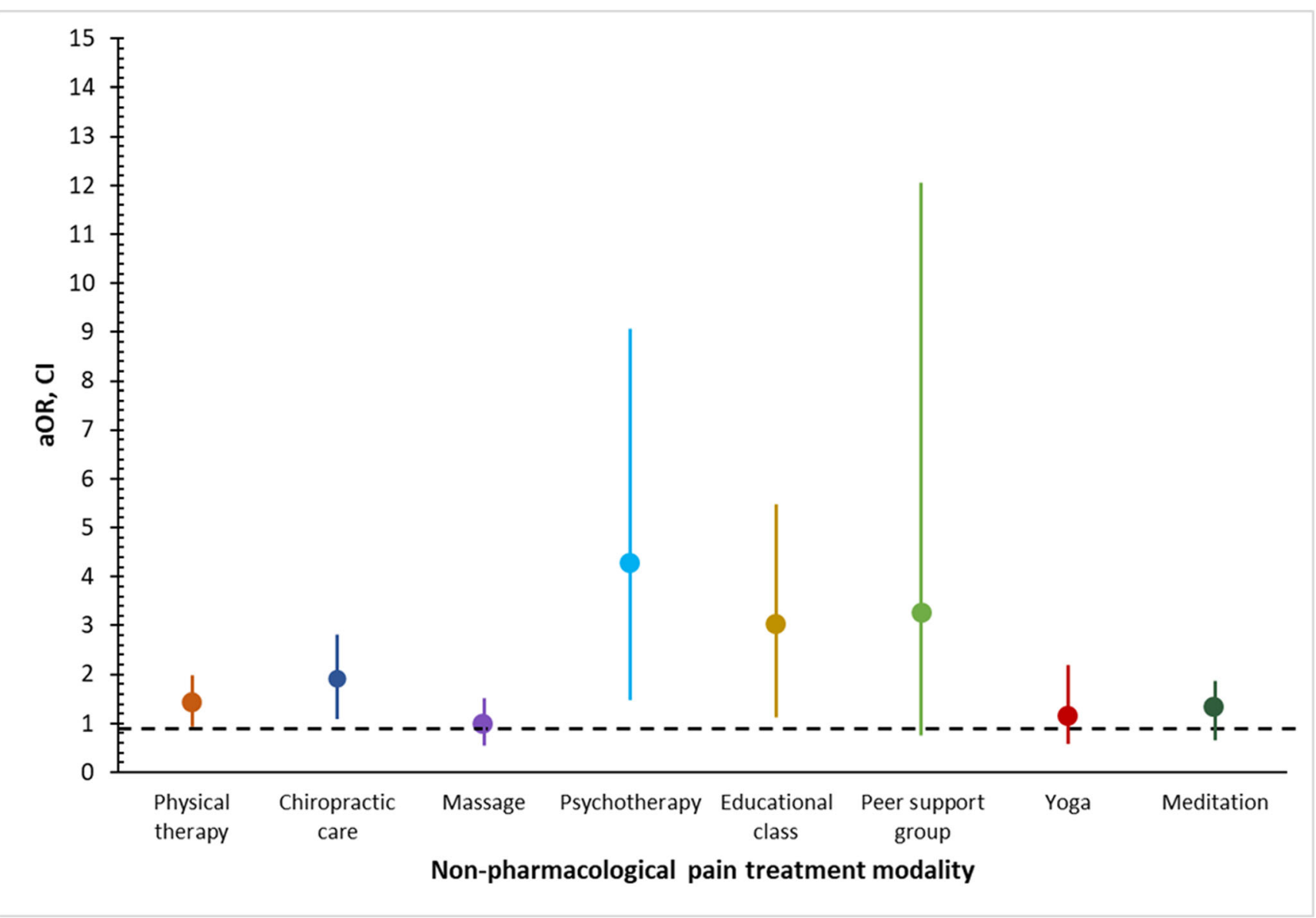

Figure 1 Adjusted odds ratios (aOR) for associations of VHA use and each NPM. Note. Lines correspond to 95\% CI around the aOR. Bars not overlapping with 1.00 indicate significant association. VHA, Veterans Health Administration; NPM, non-pharmacological treatment modalities. Models adjusted for age, sex, race/ethnicity, education, family income, US region, urbanicity, generalized anxiety disorder symptoms, major depressive disorder symptoms, BMI, and use of cigarettes or prescription opioids.

within the past 12 months. Nearly $33 \%$ of veterans had chronic pain, and $19.1 \%$ had high-impact chronic pain.

Compared to non-VHA veterans, VHA patients were more likely to be racial/ethnic minorities (38.3\% vs. $18.7 \%)$, have lower income ( $64.4 \%$ vs. $49.6 \%)$, and have moderate/severe symptoms of GAD (10.1\% vs. $3.4 \%)$ or MDD (12.4\% vs. $6.1 \%$ ). VHA patients were also more likely than non-VHA veterans to have chronic pain $(45.2 \%$ vs. $26.8 \%)$ and highimpact chronic pain $(26.0 \%$ vs. $15.2 \%$; Table 1$)$.

\section{Pain Treatment}

Among veterans with chronic pain, approximately $14.9 \%$ used prescription opioids, $43.8 \%$ used any NPM, and $16.4 \%$ used multimodal NPM. The most common NPM for pain were physical therapies $(21.1 \%)$, massage $(15.3 \%)$, relaxation strategies $(13.3 \%)$, and chiropractic care $(11.4 \%)$. VHA patients were more likely than non-VHA veterans to use any NPM ( $49.8 \%$ vs. $39.4 \%)$, or multimodal NPM (23.2\% vs. $16.4 \%)$. Compared to non-VHA veterans, VHA patients were also more likely to use physical therapies $(24.4 \%$ vs. $18.3 \%)$, chiropractic care (14.1\% vs. $9.3 \%)$, an educational class/ workshop ( $8.3 \%$ vs. $3.5 \%$ ), meditation/relaxation strategies
(16.0\% vs. $11.0 \%)$, yoga/tai chi (7.2\% vs. $4.9 \%)$, and psychotherapy $(7.1 \%$ vs. $1.5 \%$; Table 2$)$.

\section{Adjusted Associations Between VHA Utilization and NPM}

Compared to non-VHA veterans with chronic pain, VHA patients were more likely to use any NPM $(\mathrm{aOR}=1.52$, 95\% CI: 1.07-2.16; Table 3). VHA veterans were also more likely than non-VHA veterans to use multimodal NPM (aOR $=1.80,95 \%$ CI: $1.12-2.87$ ) than no NPM (Table 4). Among veterans with chronic pain, VHA veterans were more likely to use chiropractic care $(\mathrm{aOR}=1.90,95 \% \mathrm{CI}$ : $1.12-3.22)$, educational class/workshop $(\mathrm{aOR}=3.02,95 \%$ CI: $1.35-6.73)$, or psychotherapy (aOR $=4.28,95 \%$ CI: $1.69-10.87$; Fig. 1$)$.

\section{DISCUSSION}

In a nationally representative survey of the US population, we examined differences in chronic pain and NPM use among military veterans by their use of VHA healthcare. In this study, VHA patients demonstrated greater prevalence of chronic pain 
and moderate-severe symptoms of anxiety and depression compared to non-VHA veterans. Importantly, among veterans with chronic pain, VHA patients were also more likely than non-VHA veterans to use NPM, including chiropractic care, psychotherapy, an educational class/workshop, or multimodal pain treatment. These results suggest that the VHA is an important resource and possible facilitator of NPM. These findings may help inform healthcare delivery practices of non-VHA healthcare systems that are facing challenges incorporating evidence-based NPM into routine care.

Over $45 \%$ of VHA patients had chronic pain and $25 \%$ had high-impact chronic pain. These rates are even higher than those found in previous national studies, possibly due to the aging of former combat veterans susceptible to chronic pain ${ }^{53}$ or increasing national rates of chronic pain ${ }^{43}$ and the comorbidities that exacerbate it. ${ }^{54}$ Furthermore, VHA patients were disproportionately affected by psychiatric symptoms and cigarette use. The burden caused by high-impact chronic pain and psychiatric symptoms among VHA patients could explain their greater use of NPM, as this group may be seeking treatment for these morbidities. Epidemiological research demonstrates a bi-directional association between chronic pain, and psychiatric or substance use disorders, ${ }^{8}$ though reduction in pain intensity with psychosocial interventions can reduce the severity of substance use and mental health symptoms. ${ }^{55,56}$ NPM offer treatment for the physical (e.g., strength and flexibility), psychological (e.g., self-efficacy and management of catastrophizing), and social (e.g., social isolation and unemployment) consequences of chronic pain that perpetuate its health comorbidities among VHA patients. ${ }^{57}$ Therefore, continued attempts to expand NPM in among VHA patients may assist with reducing their high physical and psychiatric burden.

In this study, NPM use was more common among VHA patients, a group with significantly fewer economic resources than non-VHA veterans. Among non-VHA patients, insurance limits for NPM are common, ${ }^{21}$ and therapies such as acupuncture, massage, and mind-body interventions (i.e., yoga and tai chi) are not routinely covered, while psychotherapies for pain conditions are not reimbursable in most states. ${ }^{22}$ Despite an almost universal lack of insurance coverage for many NPM in the US, expansion of coverage for these therapies increases NPM use ${ }^{58}$ and provides long-term cost-saving benefits. ${ }^{59,60}$ Further empirical support for the financial and health benefits of NPM expansion might help overcome existing reimbursement constraints. In addition, transportation difficulties and greater distance to clinics that offer NPM may limit use of these services. ${ }^{19,}{ }^{20}$ Use of telemedicine in VHA and non-VHA health systems has increased due to the COVID-19 pandemic ${ }^{61,62}$ and may be useful for overcoming challenges in providing NPM, particularly for patients in rural areas where NPM access is limited by provider shortages, appointment availability, and number of specialty pain clinics. ${ }^{63}$ In this sample, women, younger adults, and veterans with higher educational attainment were also more likely to use NPM. These findings are like those of a prior VHA study, ${ }^{64}$ underscoring the need to expand NPM to sociodemographic groups less likely to use these services.

Treatment preferences, provider beliefs about pain treatment, and VHA efforts to improve access to NPM may explain the higher rates of these services among VHA patients. Historically, VHA patients were unenthusiastic about NPM. ${ }^{19}$ However, as prescribed opioids for chronic pain management are now discouraged, patient interest in NPM may be increasing. ${ }^{41,65}$ VHA providers now also prefer psychotherapy and physical therapy interventions for chronic pain. ${ }^{66} \mathrm{VHA}-$ implemented clinical and research initiatives have likely also expanded NPM use among veterans with chronic pain. For example, the VHA National Pain Management Strategy has advanced a biopsychosocial approach to chronic pain treatment. ${ }^{36}$ This program has informed the Pain Management Collaboratory, an $\$ 88$ million initiative of the NIH and VA/ DoD that has reinforced NPM research and clinical implementation. ${ }^{35}$ The VHA Whole Health Program has also increased dissemination of NPM, ${ }^{65}$ and access to NPM may further improve under the Maintaining Internal Systems and Strengthening Integrated Outside Networks (MISSION) Act. ${ }^{67,68}$

Study limitations are noted. First, because the 2019 NHIS was the first version to assess NPM, data were cross-sectional, and we cannot determine the temporality of the associations. Second, our measure of NPM was limited to use within the past 3 months, and we were unable to examine frequency or duration of NPM use or whether VHA patients received NPM from non-VHA providers. Third, chronic pain was determined via self-report and NHIS did not have numerical ratings of pain intensity. However, our definition of chronic pain was consistent with the definition provided by the International Association for the Study of Pain, ${ }^{1,2}$ and high-impact chronic pain was used to approximate pain severity. Fourth, symptoms of posttraumatic stress disorder, use of alcohol or illicit substances, and non-opioid medications for pain (e.g., nonsteroidal anti-inflammatory drugs [NSAIDS], gabapentinoids, serotonin and norepinephrine reuptake inhibitors [SNRIs]) were not assessed in the 2019 NHIS, and therefore no conclusions can be drawn regarding their association with NPM. Fifth, this was a sample of veterans, who are largely white males of middle age or older, many of whom have service-connected medical disabilities. Of these, VHA patients receive pain services regardless of insurance type (e.g., CHAMPVA, TRICARE) and may qualify for subsidized services despite limited income. Therefore, our findings may not be generalizable to other patient populations, including those that experience insurance constraints for NPM. Despite these limitations, this investigation had notable strengths. This study had a sufficient sample size to control for many health and sociodemographic confounders. ${ }^{69}$ Moreover, the 2019 NHIS is a rich data source to examine many NPM. Finally, we examined the prevalence of chronic pain and NPM between VHA 
patients and non-VHA veterans, which is an important advance over most pain studies of veterans that often include only VHA patients.

Studies examining access to NPM for chronic pain are important to inform clinical practice, particularly as prescriptions for opioids wane while chronic pain patients continue to require care. Our study demonstrated that VHA patients, a population highly burdened by chronic pain and its comorbidities, were more likely than non-VHA veterans to use psychotherapy, chiropractic care, educational classes/workshops, or multimodal NPM. This study supports previous findings suggesting that VHA initiatives have expanded use of NPM. ${ }^{40}$ Future studies should assess longitudinal changes in NPM since enactment of the $\mathrm{CDC}$ and VA/DoD opioid prescribing guidelines in 2016-2017, and the influence of the COVID-19 pandemic on NPM use. Furthermore, implementation research is needed to determine how NPM can best be disseminated in other health systems. Perhaps most importantly, future research should aim to understand how the shift away from prescription opioids towards NPM has affected population health and well-being, e.g., pain, morbidity, and mortality. Studies are needed to ascertain rates of NPM use among VHA patients deprescribed long-term prescription opioids, and whether use of NPM can offer health benefits to existing opioid taper protocols or after tapers have ended. Such research has important health implications for patients susceptible to the consequences of untreated chronic pain and opioid discontinuation. ${ }^{70,71}$

Corresponding Author: Deborah S. Hasin, PhD; New York State Psychiatric Institute, 1051 Riverside Drive, , Box 123New York, NY 10032, USA (e-mail: dsh2@cumc.columbia.edu).

Funding This study received funding from the National Institute on Drug Abuse (NIDA; grant number RO1DA048860) and the NIDA Substance Abuse Epidemiology Training Program (grant number 5T32DA031099).

\section{Declarations:}

Conflict of Interest: KMK and AJS provide expert testimony for various law firms in the US. AJS receives royalties from UptoDate Inc. and serves on Advisory Boards for Indivior Inc. Alkermes Inc., and the PTSD and Substance Abuse (PASA) DSMB. DSH receives funding from Syneos Health for an unrelated project on the measurement of opioid addiction among chronic pain patients.

Open Access This article is licensed under a Creative Commons Attribution 4.0 International License, which permits use, sharing, adaptation, distribution and reproduction in any medium or format, as long as you give appropriate credit to the original author(s) and the source, provide a link to the Creative Commons licence, and indicate if changes were made. The images or other third party material in this article are included in the article's Creative Commons licence, unless indicated otherwise in a credit line to the material. If material is not included in the article's Creative Commons licence and your intended use is not permitted by statutory regulation or exceeds the permitted use, you will need to obtain permission directly from the copyright holder. To view a copy of this licence, visit http://creativecommons. org/licenses/by/4.0/.

\section{REFERENCES}

1. Treede RD, Rief W, Barke A, et al. A classification of chronic pain for ICD-11. Pain. 2015;156(6): 1003.

2. Treede RD, Rief W, Barke A, et al. Chronic pain as a symptom or a disease: the IASP Classification of Chronic Pain for the International Classification of Diseases (ICD-11). Pain. 2019;160(1):19-27.

3. Yong RJ, Mullins PM, Bhattacharyya N. Prevalence of chronic pain among adults in the United States. Pain. 2021. doi: https://doi.org/10. $1097 /$ j.pain.0000000000002291. Epub ahead of print.

4. Gaskin DJ, Richard P. The economic costs of pain in the United States. J Pain. 2012;13(8):715-24.

5. Dahan A, van Velzen M, Niesters M. Comorbidities and the complexities of chronic pain. Anesthesiology. 2014;121(4):675-7.

6. Fine PG. Long-term consequences of chronic pain: mounting evidence for pain as a neurological disease and parallels with other chronic disease states. Pain Med. 2011;12(7):996-1004.

7. Oliveira CB, Maher CG, Franco MR, et al.Co-occurrence of chronic musculoskeletal pain and cardiovascular diseases: a systematic review with meta-analysis. Pain Med. 2020;21(6):1106-21.

8. Hooten WM. Chronic pain and mental health disorders: shared neural mechanisms, epidemiology, and treatment. Mayo Clin Proc. 2016;91(7):955-970.

9. Bernard SA, Chelminski PR, Ives TJ, et al. Management of pain in the United States-A brief history and implications for the opioid epidemic. Health Serv Insights. 2018;26(11):1178632918819440

10. Centers for Disease Control and Prevention. Atlanta, GA: CDC, National Center for Health Statistics. Available at http://wonder.cdc.gov. 2020. Accessed August 1, 2021.

11. Kolodny A, Courtwright DT, Hwang CS, et al. The prescription opioid and heroin crisis: a public health approach to an epidemic of addiction. Annu Rev Public Health. 2015;18(36):559-74.

12. Goldstick JE, Guy GP, Losby JL, Baldwin G, Myers M, Bohnert AS. Changes in initial opioid prescribing practices after the 2016 release of the CDC Guideline for Prescribing Opioids for chronic pain. JAMA Netw Open. 2021;4(7):e2116860.

13. Dowell D, Haegerich TM, Chou R. CDC guideline for prescribing opioids for chronic pain-United States, 2016. JAMA. 2016;315(15):1624-45.

14. Tick H, Nielsen A, Pelletier KR, et al.Evidence-based nonpharmacologic strategies for comprehensive pain care: the consortium pain task force white paper. Explore (NY). 2018;14(3):177-211.

15. Skelly AC, Chou R, Dettori JR, et al. Noninvasive nonpharmacological treatment for chronic pain: A systematic review update. Rockville (MD): Agency for Healthcare Research and Quality (US). 2020.

16. Herman PM, Yuan AH, Cefalu MS, et al. The use of complementary and integrative health approaches for chronic musculoskeletal pain in younger US Veterans: An economic evaluation. PloS One. 2019;14(6):e0217831.

17. Lisi AJ, Corcoran KL, DeRycke EC, et al. Opioid use among veterans of recent wars receiving Veterans Affairs chiropractic care. Pain Med. 2018;1(19); 54-60.

18. Gulliford M, Figueroa-Munoz J, Morgan M, Hughes D, Gibson B, Beech R, Hudson M. What does 'access to health care' mean? J Health Serv Res Policy. 2002;7(3):186-8.

19. Becker WC, Dorflinger L, Edmond SN, Islam L, Heapy AA, Fraenkel L. Barriers and facilitators to use of non-pharmacological treatments in chronic pain. BMC Fam Prac. 2017;18(1):1-8.

20. Giannitrapani $\mathbf{K}, \mathbf{M c C a a} \mathbf{M}$, Haverfield $\mathbf{M}$, et al. Veteran experiences seeking non-pharmacologic approaches for pain. Mil Med. 2018;183(1112):e628-34.

21. Bonakdar R, Palanker D, Sweeney MM. Analysis of state insurance coverage for nonpharmacologic treatment of low back pain as recommended by the American College of Physicians Guidelines. Glob Adv Health Med. 2019; 29(8): 1-9.

22. Heyward J, Jones CM, Compton WM, et al. Coverage of nonpharmacologic treatments for low back pain among US public and private insurers. JAMA Netw Open. 2018;5(6): e183044.

23. Bushnell MC, Frangos E, Madian N.Non-pharmacological treatment of pain: Grand challenge and future opportunities. Front Pain Res. 2021;28(2): 13 .

24. Dahlhamer J, Lucas J, Zelaya C, et al. Prevalence of chronic pain and high-impact chronic pain among adults-United States, 2016. MMWR Morb Mortal Wkly Rep. 2018;67(36):1001.

25. Centers for Disease Control and Prevention. Percentage of adults aged $\geq 20$ years who had chronic pain, by veteran status and age group - 
National Health Interview Survey, United States, 2019. MMWR Morb Mortal Wkly Rep. 2020;69:1797.

26. Teeters JB, Lancaster CL, Brown DG, Back SE. Substance use disorders in military veterans: prevalence and treatment challenges. Subst Abuse Rehabil. 2017;8:69-77.

27. Lin LA, Peltzman T, McCarthy JF, Oliva EM, Trafton JA, Bohnert AS Changing trends in opioid overdose deaths and prescription opioid receipt among veterans. Am J Prev Med. 2019; 1;57(1):106-10.

28. Seal KH, Shi Y, Cohen G, et al. Association of mental health disorders with prescription opioids and high-risk opioid use in US veterans of Iraq and Afghanistan. JAMA. 2012;307(9):940-7

29. Trivedi RB, Post EP, Sun H, et al. Prevalence, comorbidity, and prognosis of mental health among US veterans. Am J Public Health. 2015; 105(12):2564-9

30. Gellad WF, Good CB, Shulkin DJ. Addressing the opioid epidemic in the United States: lessons from the Department of Veterans Affairs. JAMA Intern Med. 2017;177(5):611-2.

31. U.S. Department of Veterans Affairs. VA reduces prescription opioid use by $64 \%$ during past eight years. Office of Public and Intergovernmental Affairs. 2020. Available at https://www.va.gov/opa/pressrel/ pressrelease.cfm?id=5492. Accessed August 1, 2021.

32. U.S. Department of Veterans Affairs/U.S. Department of Defense. VA/DoD Clinical Practice Guideline for Opioid Therapy for Chronic Pain (2017). Available at www.healthquality.va.gov/guidelines/Pain/cot/. Accessed May 2021

33. Meerwijk EL, Larson MJ, Schmidt EM, et al. Nonpharmacological treatment of army service members with chronic pain is associated with fewer adverse outcomes after transition to the Veterans Health Administration. J Gen Intern Med. 2020;35(3):775-83.

34. U.S. Department of Veterans Affairs. Veterans Benefits Administration, Compensation. 2018. Available at: https://www.benefits.va.gov/REPORTS?abr/docs/2019-compensation.pdf. Accessed May 2021.

35. Kerns RD, Brandt CA, Peduzzi P.NIH-DoD-VA pain management collaboratory. Pain Med. 2019;20(12):2336-45

36. Kerns RD, Philip EJ, Lee AW, Rosenberger PH. Implementation of the Veterans Health Administration national pain management strategy. Transl Behav Med. 2011;1(4):635-43.

37. Krejci LP, Carter K, Gaudet T. Whole health: the vision and implementation of personalized, proactive, patient-driven health care for veterans. Med Care. 2014;52:S5-8.

38. Farmer CM, Hosek SD, Adamson DM. Balancing demand and supply for veterans' health care: a summary of three RAND assessments conducted under the Veterans Choice Act. Rand Health Q. 2016;6(1):12

39. Meffert BN, Morabito DM, Sawicki DA, et al. US Veterans who do and do not utilize VA healthcare services: Demographic, military, medical, and psychosocial characteristics. Prim Care Companion CNS Disord. 2019:21(1):18m02350

40. Frank JW, Carey E, Nolan C, Kerns RD, Sandbrink F, Gallagher R, Ho PM. Increased nonopioid chronic pain treatment in the Veterans Health Administration, 2010-2016. Pain Med. 2019;20(5):869-77.

41. Taylor SL, Hoggatt KJ, Kligler B. Complementary and integrated health approaches: What do veterans use and want. J Gen Intern Med. 2019;34(7):1192-9.

42. Pitcher $\mathbf{M H}$, Von Korff M, Bushnell MC, Porter L. Prevalence and profile of high-impact chronic pain in the United States. J Pain. 2019;20(2):146-60.

43. Zajacova A, Grol-Prokopczyk H, Zimmer Z. Pain Trends Among American Adults, 2002-2018: Patterns, Disparities, and Correlates. Demography. 2021;58(2):711-38.

44. Zelaya CE, Dahlhamer JM, Lucas JW, Connor EM. Chronic pain and high-impact chronic pain among US adults, 2019. NCHS Data Brief. 2020;(390): 1-8

45. Nahin RL. Categorizing the severity of pain using questions from the 2012 National Health Interview Survey. J Pain Res. 2016;9: 105-113.

46. Kligler B, Bair MJ, Banerjea R, et al. Clinical policy recommendations from the VHA state-of-the-art conference on non-pharmacological approaches to chronic musculoskeletal pain. J Gen Intern Med. 2018;33(1):16-23.

47. Kroenke K, Strine TW, Spitzer RL, Williams JB, Berry JT, Mokdad AH. The PHQ-8 as a measure of current depression in the general population. J Affect Disord. 2009;114(1-3):163-73.

48. Spitzer RL, Kroenke K, Williams JB, Löwe B. A brief measure for assessing generalized anxiety disorder: the GAD-7. Arch Intern Med. 2006;166(10): 1092-7.

49. Chapman SL, Wu LT. Associations between cigarette smoking and pain among veterans. Epidemiol Rev. 2015;37(1):86-102.
50. Barnett PG, Hamlett-Berry $\mathbf{K}$, Sung HY, Max W. Health care expenditures attributable to smoking in military veterans. Nicotine Tob Res. 2014; 17(5):586-91.

51. Okifuji A, Hare BD. The association between chronic pain and obesity. J Pain Res. 2015;8:399-408.

52. Ingram DD, Franco SJ. 2013 NCHS Urban-Rural Classification Scheme for Counties. Vital Health Stat 2. 2014;166:1-73.

53. Higgins DM, Kerns RD, Brandt CA, et al. Persistent pain and comorbidity among operation enduring freedom/operation Iraqi freedom/operation new dawn veterans. Pain Med. 2014; 15(5): 782-790.

54. Seal KH, Metzler TJ, Gima KS, Bertenthal D, Maguen S, Marmar CR. Trends and risk factors for mental health diagnoses among Iraq and Afghanistan veterans using Department of Veterans Affairs health care, 2002-2008. Am J Public Health. 2009; 99(9): 1651-1658.

55. Ilgen MA, Bohnert AS, Chermack S, et al. A randomized trial of a pain management intervention for adults receiving substance use disorder treatment. Addiction. 2016;111(8):1385-93.

56. Ilgen MA, Coughlin LN, Bohnert AS, et al. Efficacy of a psychosocial pain management intervention for men and women with substance use disorders and chronic pain: a randomized clinical trial. JAMA Psychiatry. 2020;77(12): 1225-34.

57. Baria AM, Pangarkar S, Abrams G, Miaskowski C. Adaption of the biopsychosocial model of chronic noncancer pain in veterans. Pain Med. 2019;20(1): 14-27.

58. Choo EK, Charlesworth CJ, Gu Y, Livingston CJ, McConnell KJ. Increased use of complementary and alternative therapies for back pain following statewide Medicaid coverage changes in Oregon. J Gen Intern Med. 2021;36(3):676-682.

59. Andronis L, Kinghorn P, Qiao S, Whitehurst DG, Durrell S, McLeod H.Cost-effectiveness of non-invasive and non-pharmacological interventions for low back pain: a systematic literature review. Appl Health Econ Health Policy. 2017;15(2):173-201.

60. Herman PM, Anderson ML, Sherman KJ, Balderson BH, Turner JA, Cherkin DC.Cost-effectiveness of mindfulness-based stress reduction vs cognitive behavioral therapy or usual care among adults with chronic low-back pain. Spine. 2017;42(20):1511.

61. Demeke HB, Merali S, Marks S, et al. Trends in use of telehealth among health centers during the COVID-19pandemic-United States, June 26November 6, 2020. MMWR Morb Mortal Wkly Rep. 2021;70(7):240.

62. Rosen CS, Morland LA, Glassman LH, et al. Virtual mental health care in the Veterans Health Administration's immediate response to coronavirus disease-19. Am Psychol. 2021;76(1):26-38.

63. Lum HD, Nearing K, Pimentel CB, Levy CR, Hung Ww. Anywhere to anywhere: use of telehealth to increase health care access for older, rural veterans. Public Policy Aging Rep. 2020;30(1):12-8.

64. Edmond SN, Becker WC, Driscoll MA, et al. Use of nonpharmacological pain treatment modalities among veterans with chronic pain: results from a cross-sectional survey. J Gen Intern Med. 2018; 33(1):54-60.

65. Gaudet T, Kligler B. Whole health in the whole system of the veterans administration: how will we know we have reached this future state?. J Altern Complement Med. 2019;25(S1):S7-11.

66. Belitskaya-Levy I, Clark JD, Shih MC, Bair MJ. Treatment preferences for chronic low back pain: Views of veterans and their providers. J Pain Res. 2021;14:161-171.

67. Mattocks KM, Kroll-Desrosiers A, Kinney R, Elwy AR, Cunningham KJ, Mengeling MA. Understanding VA's use of and relationships with community care providers under the MISSION Act. Med Care. 2021;59(Suppl 3):S252-S258.

68. Rieselbach RE, Epperly T, Nycz G, Shin P. Community health centers could provide better outsourced primary care for veterans. J Gen Intern Med. 2019;34(1): 150-3.

69. Bujang MA, Sa'at N, Bakar TM. Sample size guidelines for logistic regression from observational studies with large population: emphasis on the accuracy between statistics and parameters based on real life clinical data. Malays J Med Sci. 2018; 25(4):122-130.

70. Agnoli A, Xing G, Tancredi DJ, Magnan E, Jerant A, Fenton JJ. Association of dose tapering with overdose or mental health crisis among patients prescribed long-term opioids. JAMA. 2021;326(5):411-9.

71. Oliva EM, Bowe T, Manhapra A, et al. Associations between stopping prescriptions for opioids, length of opioid treatment, and overdose or suicide deaths in US veterans: observational evaluation. BMJ. 2020;368:m283.

Publisher's Note: Springer Nature remains neutral with regard to jurisdictional claims in published maps and institutional affiliations. 